Revista

\title{
Multi-Ensayos
}

Vol. 6, núm. 11

ISSN: 2412-3285

https://multiensayos.unan.edu.ni

DOI: https://doi.org/10.5377/multiensayos.v6i11.9286

\section{Las Finanzas Personales un instrumento de desarrollo socio económico de los estudiantes de la FAREM-Estelí}

\section{Personal Finance is an instrument of socio-economic development of students of FAREM-Estelí}

Martha Lorena Montoya Orozco ${ }^{1}$

Recibido: 27 de octubre de 2019. Aceptado: 13 de enero de 2020

\section{RESUMEN}

Este ensayo nace como parte de las estrategias desarrolladas por la Facultad Regional Multidisciplinaria, Estelí, en la promoción de escritura académica de parte de los docentes. La temática abordada está dentro de la asignatura Finanzas I, abordando específicamente el tema de Finanzas personales como un instrumento de desarrollo socio económico de los estudiantes de la FAREM-Estelí, haciendo énfasis en los en la gestión de la economía del individuo como son el presupuesto, los ingresos, los gastos, el ahorro y la importancia de la educación financiera como pilar del desarrollo personal y su relación con el entorno así como también se exploran las consecuencias de la poca educación financiera que es ahora preocupación a nivel mundial. Se determina el concepto de finanzas personales y las diferencias con las finanzas corporativas y se habla del porqué del resurgimiento de las finanzas personales.

Palabras claves: finanzas personales; desarrollo socio económico; estudiantes.

\section{ABSTRACT}

This essay emerged as part of the strategies to promote teachers' academic writing in the Regional Multidisciplinary Faculty, Estelí. The topic addressed in this document has to do with Finance I. It discusses the issue of personal finances as an instrument of socio-economic development for students of FAREMEstelí. It emphasizes the management of individuals' economies such as budget, income, expenditure, savings, and the importance of financial education as a pillar of personal development and its relationship to the environment as well as exploring the consequences of the low financial education that is now a concern at the global level.

Keywords: personal finance; socio-economic development; students.

1 Docente UNAN-Managua/FAREM-Estelí. Correo electrónico: marthamontoya07@hotmail.com. (c) 2020 Revista Multi-Ensayos. 


\section{INTRODUCCIÓN}

Las finanzas personales es la aplicación de los principios de las finanzas a las decisiones monetarias de un individuo o una unidad familiar. Se ocupa de las formas en que los individuos o las familias obtienen su presupuesto, ahorran y gastan recursos monetarios a través del tiempo, teniendo en cuenta diversos riesgos financieros y los acontecimientos futuros de su vida.

Uno de los grandes problemas en la sociedad en general es que no existen mecanismos que aseguren una educación financiera, las personas al no tener una buena educación financiera gastan su dinero, se endeudan y dan su tiempo y libertad a terceras personas sin aprovechar sus ingresos para progresar, una persona bien informada y educada financieramente puede tomar mejores decisiones financiera y económicas en el trabajo, el hogar o en su propia comunidad, es ahí donde surge la necesidad de analizar la implicancia que tiene el fortalecer el área de finanzas personales en la comunidad estudiantil de FAREM-Estelí.

En el proceso de búsqueda se evidenció que los documentos que tratan el tema de manera global e incluyente son muy pocos, y que si bien la mayoría de ellos son conceptos emitidos por académicos, son también el producto de su punto de vista personal. Así mismo, que los que tratan en profundidad alguno de los temas de finanzas personales lo hacen de manera específica y enfocada a un tópico determinado, lográndose en el trabajo investigativo recopilar alguna información de valor académico de lo referente a las inversiones y al presupuesto principalmente, lo que hace necesario hacer una investigación profunda y desarrollar un documento que abarque todos los temas relacionados con las finanzas personales desde el punto de vista académico.

\section{DESARROLLO}

Para el desarrollo del presente ensayo se tomaron en cuenta los siguientes aspectos: Finanzas Personales y desarrollo socio económico.

Las finanzas, que se definen como el arte y la ciencia de administrar dinero, y el ser humano, enmarcado en el ser como persona económicamente activa que recauda dinero y lo gasta o lo invierte (Gitman, 2007), tienen una relación directa que es independiente de sus deseos, dado que es inherente a el devenir diario de la actividad normal, pues el individuo trabaja, gana dinero, come, compra, necesita vestido, salud, servicios, etc., y además desea su bienestar y felicidad.

Si bien existe amplia y suficiente literatura sobre este tema, es escasa la información de carácter investigativo y de autores relevantes. Gran parte de esta información no es de tipo técnico ni es el producto de un ejercicio investigativo riguroso. Esto convierte el presente estudio en un ejercicio pertinente y de gran utilidad, toda vez que tiene como propósito la recopilación de literatura de valor investigativo. (García López, 2016), El término finanzas personales, que se ha desarrollado en épocas relativamente recientes como una rama de las finanzas, a pesar de su importancia, aún no se ha universalizado dentro 
del conocimiento académico, donde más que un concepto es una definición, e incluso ha sido un tema de poca relevancia dentro de los programas de desarrollo de la asignatura de Finanzas.

Es importante también destacar que en la sociedad en general hay muy poca información referente a la educación financiera, la cual ha sido definida por la Organización para la Cooperación y el Desarrollo Económicos (OCDE) como "el proceso por el cual los consumidores financieros/inversionistas mejoran su entendimiento de productos financieros, conceptos y riesgos, y a través de la información, instrucción y/o consejos objetivos, desarrollan las habilidades y confianza para volverse más conscientes de los riesgos financieros y las oportunidades, para tomar decisiones informadas, para conocer dónde acudir para obtener ayuda, y para tomar otras acciones efectivas para mejorar su bienestar financiero." (Educación Financiera para Jóvenes: Una visión introductoria, 2013)

Las finanzas personales se componen de múltiples conceptos, dentro de los cuales, en la literatura los más relevantes incluyen el presupuesto, los ingresos, los gastos y el ahorro.

El primer elemento es el presupuesto, que se define como el proceso de planificación financiera inicia con los planes a largo plazo, los estratégicos, y estos, a su vez, dirigen la formulación de los planes y presupuestos a corto plazo, u operativos. Generalmente los planes y presupuestos a corto plazo llevan a cabo los objetivos estratégicos de largo plazo (Zutter, 2007), sin embargo se ha logrado identificar que pese a los conocimientos en presupuesto existente los estudiantes no utilizan este instrumento para proyectar sus finanzas.

El segundo elemento dentro de los tópicos de finanzas personales se refiere o a todas las entradas de dinero que tienen lugar en la unidad familiar o personal, en un horizonte de tiempo determinado, que abarca el presupuesto. Estos ingresos pueden originarse en sueldos, negocios, pensiones, subsidios, giros de terceros. En el caso del presupuesto anual, se tendrán en cuenta primas, rendimientos financieros, dividendos de acciones y devolución de impuestos. (Aibar Ortiz, 2012), de los cuatros elementos abordados este es el que mejor conocimiento poseen los estudiantes ya que están claros cuáles son sus ingresos en los diferentes periodos de tiempo.

En el tercer ítem se abordan los gastos, Este concepto se define así: "Los gastos son todas las salidas de dinero, que se producen durante el periodo que abarca el presupuesto que realiza cualquier miembro de la unidad familiar" (Aibar Ortiz, 2012), este elemento es el que representa mayor dificultad ya que la mayoría de la población en general expresa desde nuestras vivencias diarias, que sus gastos son mayores a los ingresos, convirtiéndose en un aspecto clave para gestionar en el manejo y planeación de estrategias destinadas a mejorar las finanzas personales

El cuarto elemento tiene que ver con el ahorro, el cual se define como Ahorrar es tomar una porción del ingreso no destinada al consumo, y conservarla en cualquier ente económico empresarial o personal para ser utilizado en un futuro. Antiguamente, las transacciones se hacían mediante el trueque. En esa época, las personas valoraban más los bienes que mantenían sus características con el paso del tiempo, y así preservaban la riqueza, y los ahorros estaban dados por estos bienes, pero no incrementaban su valor 
con el paso del tiempo. Posteriormente, aparece el dinero, con el que ahora se podían comprar otros bienes, los cuales 34 podían guardarse para intercambios futuros. $Y$ en caso de no poder invertirlo, se debía guardar para ser usado después, pero sin aumentar su valor. (Gloria Rivera, 2012), sin embargo se ha logrado identificar que no existe una cultura de ahorro en los estudiantes, ya que la mayoría de los ingresos son gastados en el periodo en que son devengados.

El nivel o estatus socioeconómico es una medida total económica y sociológica combinada de la preparación laboral de una persona y de la posición económica y social individual o familiar en relación a otras personas, basada en sus ingresos, educación, y empleo. Al analizar el nivel socioeconómico de una familia se analizan, los ingresos del hogar, los niveles de educación, y ocupación, como también el ingreso combinado, comparado con el individual, y también son analizados los atributos personales de sus miembros.

El desarrollo socio económico se entiende como las actividades en que los factores sociales y económicos, se traducen en el crecimiento de la economía y la sociedad y que son medibles, de lo anterior podemos determinar que una debida estrategia de educación de las finanzas personales puede incidir positivamente en el desarrollo socio económico, seleccionando a los estudiantes de la FAREM Estelí como el primer nivel de incidencia y desde donde podemos realizar otras estrategias de educación financiera al resto de la población.

\section{CONCLUSIÓN}

Las finanzas personales existen desde hace varios siglos, casi desde que existe el dinero, pero no se habían definido como tales, y desde ese entonces habían sido aplicadas de manera inadvertida por la población en general, pues todos tenemos que ver con el dinero en el día a día.

Las finanzas personales entendidas como el manejo de los recursos por parte del individuo no se han incorporado aún en los procesos formativos. Esto lleva a gestionar de manera inadecuada dichos recursos, que generalmente son escasos. Un obstáculo para el acceso a esta formación y al ejercicio adecuado en este sentido es que el ser humano tiene como fin último la felicidad, y la busca activamente. Esto evita que se concentre en la obtención del conocimiento en lo referente a finanzas personales; incluso, a veces, aunque el individuo lo tenga, antepone la búsqueda de la felicidad y la satisfacción a la gestión.

\section{REFERENCIAS}

Aibar Ortiz, M. J. (2012). Finanzas personales: planificación, control y gestión. Espana. GARCÍA, J. C. (2016). LAS FINANZAS PERSONALES, UN CONCEPTO QUE VA MÁS ALLÁ DE SU ESTRUCTURA. Pereira, Colombia.

Gitman, L. J. (2007). Principios de administración financiera. Naucalpan de Juárez: juarez.

Gloria Rivera, R. A. (2012). Finanzas personales encaminadas. UNAM, Dirección General de Bibliotecas, tesis. 
Instituto Universitario de Analisis Economico y Social. (2013). Educación Financiera para Jóvenes: Una visión introductoria.

jovenes, E. F. (2013). Una Vision Introductoria.

Zutter, G. y. (2007). 